\title{
跨期决策是否基于“折扣计算”：来自双分离范式的 证据
}

张阳阳 ${ }^{1}$, 周蕾 $2,3,4$, 游旭群 ${ }^{1}$, 李纾 ${ }^{2,3^{*}}$, 梁竹苑 $2,3^{*}$

1. 陕西师范大学心理学院, 西安 710062 ;

2. 中国科学院心理研究所中国科学院行为科学重点实验室, 北京 100101 ;

3. 中国科学院大学心理学系, 北京 100049 ;

4. 暨南大学管理学院, 广州 510632

*联系人, E-mail: lishu@psych.ac.cn; liangzy@psych.ac.cn

收稿日期：2018-01-30; 接受日期：2018-03-30; 网络版发表日期：2018-05-15

国家自然科学基金(批准号: 71471171, 71701117, 31471005)、中国博士后科学基金(批准号: 2017M613041)和中国科学院行为科学重点实验室 自主研究课题(批准号: Y5CX052003)资助

摘要跨期决策指对发生在不同时间点上的结果之间进行的权衡. 跨期决策主要理论可依据跨期决策是否基于 折扣计算分为两类: 折扣模型和非折扣模型，但目前学界却缺乏研究直接检验跨期决策的折扣计算假设是否成 立. 因此，本研究通过设计新的实验范式检验了跨期决策的“折扣计算”假设。该范式对比了符合折扣计算假设的 基线折扣任务和被试自主进行跨期决策的自主跨期任务的任务表现和分层贝叶斯模型拟合结果，并基于双分离 逻辑，分别选择了仅影响基线折扣任务或者自主跨期任务的操作变量(计算难度或选项的结果大小)与调节变量 (计算能力或认知反思风格). 研究结果发现，基线折扣任务的任务表现符合折扣计算假设的预测: 其反应时随计 算难度上升而增加，且其正确率受到计算难度和计算能力的影响; 自主跨期决策任务的任务表现与基线折扣任务 不同: 其反应时短于基线折扣任务, 且当选项结果越大, 其选择SS选项的比例越少; 调节变量和模型拟合出现了双 分离效应，计算能力只影响基线折扣任务中的选择，认知反思风格只影响自主跨期任务偏好选择; 计算难度只影 响基线折扣任务的模型拟合度，而选项结果大小只影响自主跨期任务的模型拟合度. 本研究结果不仅证明了新实 验范式在检验折扣计算假设中的有效性，且从任务表现、调节变量和模型拟合层面看，自主跨期任务可能并不符 合折扣计算假设的预测. 本研究为更好地检验跨期决策的过程提供了新的方法学上的有益探索.

关键词 跨期决策, 折扣计算, 基线折扣任务, 双分离, 分层贝叶斯模型

跨期决策(intertemporal decision-making)指对发生 在不同时间点上的结果之间进行的权衡 ${ }^{[1]}$. 跨期决策 可以涉及收益，如获得一笔奖金后是现在立刻花掉还
是暂时存在银行里; 也可以涉及损失，如是否给自己 的爱车买上一份保险，来降低以后可能承担的损失. 跨期决策可以小至个体的日常生活和经济行为，也可

引用格式: 张阳阳, 周蕾, 游旭群, 等. 跨期决策是否基于“折扣计算”: 来自双分离范式的证据. 中国科学: 生命科学, 2018, 48: 592-608 Zhang Y Y, Zhou L, You X, et al. Is intertemporal decision-making based on a temporal discounting process? Evidence from double-dissociation paradigm (in Chinese). Sci Sin Vitae, 2018, 48: 592-608, doi: 10.1360/N052017-00104 
以大到一个公司或者国家的策略制定, 如是否加强环 境保护减少过度开发, 从而保持可持续发展等. 正如 Adam Smith指出的, 跨期决策不仅影响一个人的健康 财富与幸福, 也影响一个国家的繁荣和昌盛 ${ }^{[2]}$. 与跨期 决策在人类社会中的重要性相悖, 目前学界对跨期决 策的主流理论和基本度量指标涉及的基本假设, 尚缺 少一致的观点.

跨期决策主要理论可依据跨期决策是否基于折扣 计算分为两类: 折扣模型和非折扣模型. 以折扣效用模 型和双曲线折扣模型为主的折扣家族模型认为, 决策 者是根据时间折扣函数和主观价值函数, 计算出每个 选项的折扣值, 然后比较并选择折扣值较大的选项. 而非折扣模型认为, 决策者受限于认知加工能力等因 素的影响, 不存在如折扣模型所假设的折扣计算过程. 因此跨期决策是否基于折扣计算过程, 是折扣模型和 非折扣模型的主要分歧点. 然而, 由于前人研究范式 和方法等的问题, 跨期决策的折扣计算假设尚未得到 直接有效的检验.

因此, 本研究设计了新的实验范式, 结合了双分离 逻辑和分层贝叶斯建模, 从反应时、选择结果、个体 差异变量和模型检验层面, 为直接检验跨期决策的折 扣计算假设提供更加充分的证据, 力求为跨期决策研 究提供新的研究方法和思路.

\section{1 跨期决策的折扣模型与非折扣模型}

跨期决策的主流理论均具有一个共同的基本隐含 假设, 即跨期决策遵循折扣计算的过程. 这类模型中最 早的代表是诺贝尔经济学奖获得者Samuelson ${ }^{[3]}$ 在 1937年提出的折扣效用模型(discounted-utility model, DU). 该模型认为, 人们将按照同一比率(时间折扣率) 对未来不同时间点的效用进行折扣，未来各时期折扣 效用之和是决策的总效用.

随后, 研究者在DU模型的基础上发展了其他折扣 理论模型, 如双曲线(hyperbolic)折扣模型 ${ }^{[4]}$ 和准双曲 线(quasi-hyperbolic)折扣模型 ${ }^{[5]}$ 等. 这些理论均假设, 决策者基于时间对每个延迟结果进行折扣, 并依据折 扣后的结果为每个选项计算一个总分, 决策者会选择 或者偏爱“较大”总分的选项. 如最常见的一种双曲线 折扣模型可用公式 1 描述 ${ }^{[4]}$.

$$
F(d)=\frac{1}{1+k d},
$$

$F(d)$ 是个体的折扣函数, $d$ 表示未来的某个时间点, $k$ 表 示个体的时间折扣率. 一个价值在时间点 $d$ 的效用, 即 折扣效用, 会依据 $F(d)$ 而打折. $k$ 越大, 时间点 $d$ 的折扣 效用越小, 说明人们越没有耐心, 越不愿意为了未来的 利益而等待.

与折扣效用模型相比, 双曲线折扣模型并未假设 一个恒定不变的时间折扣率, 因而可以较好地解释共 同差异效应和量级效应等异象. 但从模型公式可以看 出, 尽管双曲线折扣家族模型与折扣效用模型在模型 参数和表达式上有所不同, 但二者仍共同假设决策者 存在折扣计算的认知加工过程, 即通过时间折扣率对 未来价值 $u(d)$ 进行折扣, 从而做出决策.

与跨期决策的主流理论不同, 进入 21 世纪以来, 学 者们提出了一批跨期决策的非折扣计算模型. 这些模 型主要基于 1978 年诺贝尔经济学奖得主 $\operatorname{Simon}^{[6]}{ }^{\left[{ }^{\prime}\right.}$ 出 的有限理性(bounded rationality)假设, 即决策者受计算 能力等因素的限制, 不是无限理性的. 因此, 这些跨期 决策的非折扣计算模型摈弃了折扣计算过程的假设, 不寻求具有最大总效用的选项, 而是将在某个维度上 处于劣势的选项排除出局, 从而做出选择.

具体来说, 跨期决策的非折扣模型可分为两类. 其 中影响较大是基于维度比较的模型(attribute-comparison models) ${ }^{[7]}$, 该类模型的核心思想是, 认为决策者首 先对不同选项依次进行各维度之间(attribute-by-attribute)的比较, 随后基于这些维度间差异进行选择. 例 如, 权衡模型(tradeoff model)认为, 跨期决策中人们会 对时间间隔和结果差异进行权衡, 当时间间隔大于结 果差异时, 就会选择时间更早的选项, 反之则选择延 迟选项 ${ }^{[8]}$. 再如, 齐当别(equate-to-differentiate model) 抉择模型尝试将决策过程是维度间的策略应用于跨期 决策领域: 决策者处理完备择选项在一个维度(如时间 维度)间的差异之后, 再处理备择选项在另一个维度 (如结果维度)间的差异 ${ }^{[011]}$. 另一类非折扣模型则更关 注跨期决策中的认知和心理属性. 例如, 时间建构理论 (temporal construal theory)认为, 人们倾向于依据对选 项属性的不同建构水平做出跨期决策 ${ }^{[12]}$ : 对较远未来 发生事件进行抽象的高水平建构(high-level construal), 而对较近未来发生的事件进行具体的低水平建构 (low-level construal). 当选项中高建构水平的价值比低 
建构水平的价值更加正面时，选项的吸引力将随着延 迟时间的增加而增加，从而对不同时间点发生的事件 产生了不同的偏好和选择.

越来越多的实验证据从不同的角度表明，相比于 折扣模型，非折扣模型能够更好地解释人们如何进行 跨期决策. 已有大量研究对折扣模型的一些基本假设 (如独立性、可加性等)提出了质疑 ${ }^{[8,10,13]}$, 亦有研究发 现，在模型拟合的准确率上，非折扣模型的预测力优 于折扣模型 ${ }^{[14]}$. 但是, 对于折扣模型和非折扣模型的 关键分歧——跨期决策是否基于折扣计算，目前仍然 缺乏直接证据.

\section{2 跨期决策模型检验的范式和方法}

在跨期决策理论发展中，其理论模型的主要检验 方法沿袭了行为经济学的经典方法，即基于结果的模 型检验法. 其核心思想是通过检验模型是否可以解释 更多的现象，或者是否有决策现象违反模型的基本假 设，从而判断模型是否有效，并进行模型之间的优劣 的比较. 其主要方法包括结果预测(outcome prediction)与模型拟合 (model fitting). 结果预测方法主要利 用回归、方差分析等统计方法，检验决策任务的材料 参数与决策者选择结果之间的关系，判断个体实际所 做决策是否与决策模型所做的预测相符 ${ }^{[4]}$. 模型拟合 方法主要通过利用实验数据去估计代表决策过程潜在 机制的参数 ${ }^{[15]}$, 并评价模型对于个体的行为数据拟合 度的优劣 ${ }^{[16]}$.

\section{1 结果预测的模型检验法}

基于结果预测的模型检验方法在跨期决策理论发 展中起到了重要作用. 例如, 折扣模型成立的前提之一 是符合消去公理(cancellation axiom), 即人们的偏好并 不受到备择选项中共同成分的影响 ${ }^{[17]}$. 但是 $\mathrm{Rao}$ 和 $\mathrm{Li}^{[10]}$ 的研究发现, 人们在跨期决策中违背了消去公理, 从而 对跨期决策折扣求和法则提出质疑. 近年来, 又有研究 发现在损失领域的跨期决策中，存在一种折扣家族模 型所无法解释的现象: 负折扣现象 ${ }^{[18]}$. 由于这些异象 (anomalies)的出现，研究者提出了新的跨期决策理论, 如双曲线折扣模型正是因为折扣效用模型无法解释共 同差异效应(common difference effect)等异象而提出 的 ${ }^{[4]}$. 相较而言, 随后发展的准双曲线模型可以很好地 解释即刻效应(immediate effect)等 ${ }^{[5]}$. 此外, 大量非 折扣模型的检验中同样使用了结果预测和模型拟合的 方法 ${ }^{[8 \sim 10,12]}$.

虽然研究者可以通过检验折扣模型的一些假设

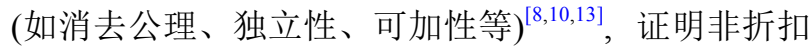
模型比折扣模型更优. 但是，当决策模型与实际选择 相悖时, 旧模型可以通过引进新的函数来优化模型的 拟合度, 这就如同希腊神话中的九头蛇一一斩去一头 生出两头, 无法从根本上证伪这些决策模型 ${ }^{[19,20]}$. 因 此，单纯地通过检验跨期决策中的异象，而不是检验 非折扣模型和折扣模型的关键分歧点, 难以从根本上 解决目前的跨期决策模型之争.

\section{2 模型拟合的模型检验法}

目前模型拟合的方法主要基于最大似然估计法 (maximum likelihood estimation, MLE). 该方法主要是 假设已知的样本满足某种概率分布, 通过寻找某个参 数使得该样本出现的概率最大, 然后将此参数作为参 数估计的真实值. 一般步骤是先对每个被试的所有试 次进行模型拟合(如折扣效用模型和双曲线模型等), 估计出每个被试的时间折扣率, 然后利用估计出的时 间折扣率对新的数据做预测，根据拟合度来判断模型 的优劣. 已有少数研究者通过模型拟合的方法对跨期 决策模型进行检验. 例如，Ericson等人 ${ }^{[14]}$ 通过此方法 发现，启发式模型(heuristic model)比传统的折扣模型 (包括折扣效用模型和双曲线模型)的拟合度更好，而 Stevens ${ }^{[21]}$ 基于最大似然估计发现, 相似性判断(similarity judgements)的模型也比传统折扣模型拥有更好的 拟合度. 但最大似然估计法存在一些弱点. 最大似然 估计法在每个被试层面估计参数，然而个体层面的参 数估计经常是充满噪音(noise)而且是不可靠的, 尤其 是在样本量小的情况下尤为明显. 如果进行群组层面 的分析(如group-level MLE), 固然可以从总体中估计 出一套参数, 从而产生更可靠的参数估计，但又忽略 了个体差异的存在.

为克服这些不足, 目前已有少数决策研究开始使 用分层贝叶斯模型(hierarchical Bayesian modeling, $\mathrm{HBM}$ )建模 ${ }^{[15,22,23]}$. 与最大似然估计法相比, 分层贝叶 斯建模具有以下优势：首先，在进行模型参数估计时， 该方法估计的是参数的后验概率分布：相对于传统的 对参数进行点估计的最大似然估计法, 能提供更丰富 
的信息, 从而避免了研究样本量小的弊端; 其次, 该方 法通过合并个体之间的信息，同时估计个体和群体层 面的参数，考虑个体差异，使得模型参数更稳定可靠; 再次, 基于贝叶斯原理, 该方法可通过计算参数后验分 布的差异, 便于进行组间比较 ${ }^{[15,22]}$. 但目前尚罕见研究 使用分层贝叶斯建模进行跨期决策模型检验.

\section{3 研究问题的提出}

从跨期决策的理论模型发展历程可见，跨期决策 是否基于折扣计算过程是跨期决策折扣模型和非折扣 模型两派理论的主要分歧点. 折扣模型认为, 决策者是 根据时间折扣函数和主观价值函数，通过加权求和计 算过程整合每个选项的所有信息，计算出每个选项的 折扣值, 然后比较并选择折扣值较大的选项. 而非折 扣模型认为，决策者受限于认知加工能力等因素的影 响，不存在折扣模型所假设的折扣计算过程. 尽管最 近的研究表明，非折扣模型可能更好地解释了个体的 跨期决策, 但是仍然缺少检验“跨期决策基于折扣计算 过程”的关键证据. 具体来说：以往研究缺乏直接证据 支持或反对跨期决策基于折扣计算假设. 以前的研究 直接检验个体的偏好选择任务是否符合某一模型的假 设 ${ }^{[4,24]}$, 或者通过脑成像或脑电数据与折扣模型中的具 体参数建立联系, 从而论证折扣模型的有效性 ${ }^{[25,26]}$. 与 风险决策类似，折扣模型总是在遇到新的挑战时就修 正折扣求和函数, 无法从根本上证伪此类模型 ${ }^{[19,27]}$. 但 针对跨期决策的基本规则，即跨期决策基于折扣计算 过程的假设，却罕见相关的直接验证. 这一困境的主 要原因之一是目前仍缺乏有效的研究范式可直接验证 跨期决策的折扣计算假设.

以往研究在模型拟合中使用的最大似然估计法有 不可避免的缺陷. 如前所述, 最大似然估计在样本量小 的时候会变得极为不可靠. 虽然这一缺陷可以通过增 加样本量来弥补, 但是其最大的缺陷一一不能兼顾群 体层面和个体差异一一依然无法直接有效地得到解 决. 通过分层贝叶斯建模将能够有效解决最大似然估 计法在模型估计中的这些缺陷.

为克服以往研究中存在的这些问题，本研究设计 了新的实验范式, 直接检验“跨期决策是否基于折扣计 算过程”的假设, 并采用了分层贝叶斯建模的方法进行 模型拟合, 力求为折扣模型和非折扣模型之争提供有
力的证据.

\section{4 研究目的与设计}

\section{1 实验范式}

为有效检验跨期决策是否基于“折扣计算”过程, 本研究设计了新的实验范式, 其中包含两个实验任务: 符合折扣计算假设的基线折扣任务和被试自主进行跨 期决策的自主跨期任务.

基线折扣任务是本实验范式中的基线任务. 该任 务情境是银行投资方案选择问题. 任务向被试呈现两 个选项，分别代表两种所需本金和投资期限均不同的 投资方案，要求被试选择出获利更大的投资方案. 鉴 于双曲线折扣模型源于银行投资计算单利的公式，银 行投资情境使被试可自主地按照双曲线折扣模型的公 式自动进行计算加工过程，整合各个方案内的投资时 间和投资本金，然后选择回报率较高的投资方案. 这 一计算过程是基于时间对金钱的“折扣计算过程”，因 此基线折扣任务符合折扣计算假设.

自主跨期任务是本实验范式中的目标任务，即跨 期决策任务中常用的两选项选择任务. 在自主跨期任 务中，被试没有决策规则的限制，任务要求被试仅根 据自身偏好，在一个延迟更短但是结果更小(sooner and smaller, SS)的选项和延迟更长但是结果更大(later and larger, LL)的选项之间做出选择. 在此任务中, 被试 按照其个人偏好或策略做出跨期决策，因此自主跨期 任务中并不包含先验的决策过程.

由上可见, 在本研究的任务范式中, 基线折扣任务 符合折扣计算假设，但自主跨期任务并不先验地假设 包含特定的决策过程. 因此，通过比较自主跨期任务 和基线折扣任务是否包含同样的心理过程，就可以直 接检验跨期决策是否基于折扣计算过程. 可以推测, 当刺激材料完全相同时，如果自主跨期任务基于折扣 计算过程，决策者在自主跨期任务和基线折扣任务中 的绩效(如反应时、选择结果等)应当无差异.

\section{2 基于双分离逻辑的研究设计}

另外, 使用了双分离(double dissociation)逻辑 ${ }^{[28,29]}$, 并参考风险决策模型检验研究 ${ }^{[30,31]}$ ，进一步检验跨期 决策的折扣计算假设. 按照双分离逻辑：如果两个任 务A和B包含同样的心理过程(在本研究中即折扣计算 
过程), “则影响被试在任务A中绩效的变量也应该影响 其在任务B中的绩效; 如果两任务包含的心理过程不 同，则在影响任务绩效的变量上应出现双分离效应, 即影响任务 $\mathrm{A}$ 的变量只对 $\mathrm{A}$ 有作用, 但对任务B无作用; 反之亦然, ${ }^{[30]}$.

根据此逻辑, 选择了两类操作变量, 分别操作了两 个实验任务中的实验材料属性. 第一类操作变量与折 扣计算过程有关，但与跨期决策自主偏好过程无关; 第二类操作变量与折扣计算过程无关，但与跨期决策 自主偏好过程有关.

具体来说, 在基线折扣任务中, 操作了选项的计算 难度. 在该任务中, 被试需要进行两阶段的计算: 首先 根据时间对选项的金钱进行折扣计算, 其次, 将计算后 的折扣选项结果进行大小比较. 根据双曲线模型(公式 1), 依据时间对金钱进行折扣时, 会将延迟选项的结果 $\mu(d)$ 与折扣函数 $F(d)$ 进行相乘的折扣计算, 然后选择具 有较大折扣效用的选项 ${ }^{[3 \sim 5]}$. 与相乘计算的难度更低 (如SS选项的结果为 $Y 50$ ) 时相比, 当相乘计算的难度 更高(如SS选项的结果为 $¥ 51$ ) 时, 折扣计算过程所需 要的认知资源更多, 认知加工深度更深, 从而会影响 基线折扣任务的任务绩效. 因此, 当选项的计算难度 相差较大, 结果的大小相差较小时(如SS选项的结果相 差 ¥ 1), 对个体的计算过程影响较大. 但在自主跨期任 务中, 如果自主跨期任务不是基于折扣计算过程, 由于 个体会比较选项的时间差异或者结果差异, 因此计算 难度可能只影响结果大小的比较过程中 ${ }^{[8,13,27]}$. 例如, 比较 $¥ 600$ 和 $¥ 723$, 以及比较 $¥ 610$ 和 $¥ 733$ 时, 由于 $¥ 610$ 的十位数位置为非 0 数字, 该非 0 数字所表征的 意义依然会被自动加工 ${ }^{[32]}$, 因而比较后者的一组结果 所需的认知加工资源更多, 可能导致其反应时更长. 所以计算难度对个体的自主跨期任务绩效影响相对基 线折扣任务更弱.

相似地, 在自主跨期任务中, 操作了选项的结果大 小. 已有大量研究发现, 在跨期决策中选项结果大小变 化会引发量级效应(magnitude effect), 即两个选项的时 间不变, 结果大小发生变化时, 个体的选择偏好也会发 生变化: 人们会表现出更多的耐心, 而选择结果更大的 选项 ${ }^{[7]}$. 因此, 假如计算难度不变, 与选项结果更小 (如 $\mathrm{SS}$ 选项的结果为 $¥ 50$ ) 时相比, 当选项结果更大(如SS 选项的结果为 $¥ 500)$ 时, 自主跨期任务中被试的选择 偏好可能发生变化. 但在基线折扣任务中, 当选项结
果相差较大(如10倍)的时, 由于两者的计算难度相差 较小(仅增加了数字的位数), 对个体的基线折扣任务 绩效影响很小. 因此, 选项的结果大小可能影响自主 跨期任务, 但可能不影响符合折扣计算过程的基线折 扣任务.

考虑到个体差异变量, 如任务相关的认知能力对 跨期决策的影响, 基于同样的双分离逻辑, 分别选择 了涉及相关认知能力的两个调节变量. 其中, 第一个 调节变量(计算能力)与折扣计算过程有关, 但与跨期 决策自主偏好过程无关; 第二个调节变量(认知反思风 格)与折扣计算过程无关, 但与跨期决策自主偏好过程 有关.

具体来说, 影响基线折扣任务的调节变量可能是 计算能力. 个体的计算能力体现了其智力水平, 能够 进行复杂的数学运算. 已有研究发现个体的计算能力 可能通过数学计算过程对风险决策产生影响 ${ }^{[30,33]}$, 计 算能力更高的个体在按照期望法则进行决策时, 其决 策的正确率也更高. 在自主跨期任务中, 选择的调节 变量为认知反思风格(cognitive reflection style $)^{[34]}$, 即 依据个体认知加工所采用的途径, 将其决策风格分为 基于反思的、较缓慢的类型 I, 和迅速得出结论而非 谨慎思考的类型 II. 已有研究发现, 个体越倾向于类 型 II 反思风格，在跨期决策中越没有耐心 ${ }^{[34]}$, 因而具 有更高的时间折扣率 ${ }^{[35]}$.

\section{3 模型拟合}

最后，尝试通过分层贝叶斯模型拟合的方法对跨 期决策的折扣计算假设进行检验. 本研究的逻辑是: 假如基线折扣任务符合折扣计算假设, 可以预期使用 折扣计算模型能够很好地拟合基线折扣任务. 如果自 主跨期任务不是基于折扣计算, 那么可以预期通过相 同折扣模型拟合自主跨期任务时，自主跨期任务的拟 合度将劣于基线折扣任务, 并且两个任务拟合后的时 间折扣率将有显著差异.

基于双分离逻辑, 可以进一步推测: 在基线任务 中, 由于任务假设被试以固定利率进行折扣计算, 可 以预期折扣模型拟合得到的时间折扣率仅受计算难度 的影响, 但不受结果大小的影响. 尤其是在低计算难度 条件下, 被试更容易依据公式进行计算, 可以预期低计 算难度条件下的拟合度要优于高计算难度条件. 由于 自主跨期任务的刺激材料和基线折扣任务完全相同, 
可以推测, 如果自主跨期任务并非基于折扣计算任务, 自主跨期任务模型拟合后的时间折扣率不受计算难度 的影响, 但受到结果大小的影响. 尤其是在小结果条件 下, 被试耗费认知资源进行折扣计算的可能性更小, 可 以预期小结果条件下的拟合度要劣于大结果条件.

\section{4 研究目的与假设}

因此, 本研究包含两个研究目的: (i) 设计一种新 的实验范式, 该实验范式中包含两类任务: 符合折扣计 算的基线折扣任务和被试自主决策的自主跨期任务; (ii) 通过比较基线折扣任务和自主跨期任务在行为绩 效和模型拟合上的差异, 检验跨期决策是否遵循折扣 计算过程. 根据双分离的实验逻辑, 本研究主要关注 反应时、选择结果、调节变量和模型拟合 4 个层面, 并基于各层面提出如下假设(表1).

反应时假设. H1a: 基线折扣任务中的反应时会受 到计算难度的影响: 计算难度越高, 反应时越长. $\mathrm{H} 1 \mathrm{~b}$ : 自主跨期任务的反应时与基线折扣任务有差异, 计算 难度对基线折扣任务中反应时的影响大于其对自主跨 期任务中反应时的影响.

选择结果假设. H2a: 基线折扣任务中的正确率(即 经过计算选择获利较多选项的比例)受到计算难度的 影响: 计算难度越高, 正确率越低. H2b: 自主跨期任务 中的选择SS选项的比例受到选项结果大小的影响, 结 果越大, 选择SS选项的比例越小.
调节变量假设. H3a: 个体的计算能力影响其基线 折扣任务的绩效: 个体的计算能力越高,在基线折扣任 务中的正确率越高. 且计算能力不影响其自主跨期任 务的绩效. H3b: 个体的认知反思风格影响其自主跨期 任务的绩效: 个体的认知反思风格越偏向直觉, 被试选 择SS选项的比例就越高, 且认知反思风格不影响其基 线折扣任务的绩效.

模型拟合假设. H4a: 使用折扣模型可以拟合基线 折扣任务, 同样的折扣模型对自主跨期任务的拟合程 度低于基线折扣任务，且两个任务拟合后得到的时间 折扣率存在差异. $\mathrm{H} 4 \mathrm{~b}$ : 计算难度和结果大小对两个任 务模型拟合的结果具有双分离效应: 计算难度仅影响 基线折扣任务的模型拟合, 且难度越小, 折扣模型对 基线折扣任务的拟合越好; 结果大小仅影响自主跨期 任务的模型拟合, 且结果越小, 折扣模型对自主跨期 任务的拟合越差.

\section{5 方法}

\section{1 被试}

通过张贴海报和网络论坛, 共招募北京市高校大 学生 31 名. 因为其中 1 名被试没能完成全部实验, 其数 据没有纳入到最后的统计分析. 最后有效被试共 30 名 (男性17名), 平均年龄(23.6 \pm 2.6 )岁. 均为右利手, 无红 绿色盲, 视力或矫正视力正常. 实验前均签署知情同 意书。

\section{表 1 研究假设和结果总结 ${ }^{a)}$}

\begin{tabular}{|c|c|c|c|c|c|}
\hline 假设 & 指标 & 操作/调节变量 & 基线折扣任务 & 自主跨期任务 & 是否支持假设 \\
\hline \multicolumn{6}{|l|}{ 反应时 } \\
\hline H1a & 反应时 & 计算难度 & $\mathrm{Y}$ & - & $\sqrt{ }$ \\
\hline $\mathrm{H} 1 \mathrm{~b}$ & 反应时 & 计算难度 & - & $\mathrm{N}$ & $\sqrt{ }$ \\
\hline \multicolumn{6}{|c|}{ 选择结果 } \\
\hline $\mathrm{H} 2 \mathrm{a}$ & 正确率 & 计算难度 & $\mathrm{Y}$ & - & $\sqrt{ }$ \\
\hline $\mathrm{H} 2 \mathrm{~b}$ & 选择偏好 & 结果大小 & - & Y & $\sqrt{ }$ \\
\hline \multicolumn{6}{|c|}{ 调节变量 } \\
\hline $\mathrm{H} 3 \mathrm{a}$ & 正确率 & 计算能力 & $\mathrm{Y}$ & $\mathrm{N}$ & $\sqrt{ }$ \\
\hline $\mathrm{H} 3 \mathrm{~b}$ & 选择偏好 & 认知反思风格 & $\mathrm{N}$ & Y & $\sqrt{ }$ \\
\hline \multicolumn{6}{|c|}{ 模型拟合 } \\
\hline $\mathrm{H} 4 \mathrm{a}$ & LOOIC指数 & 任务 & $\mathrm{Y}$ & - & $\sqrt{ }$ \\
\hline $\mathrm{H} 4 \mathrm{~b}$ & LOOIC指数 & 计算难度/结果大小 & - & Y & $\sqrt{ }$ \\
\hline
\end{tabular}

a) $\mathrm{Y}$ : 影响; N: 不影响; $\sqrt{ }$ : 支持假设; $\times$ : 不支持假设 


\section{2 实验程序与材料}

每个被试都要完成实验和问卷两个部分.

（1）实验程序. 实验为 2 (任务: 自主跨期任务, 基 线折扣任务 $) \times 2$ (计算难度: 高, 低) $\times 2$ (结果: 大, 小)的 被试内设计.

首先，实验中被试分别完成自主跨期任务和基线 折扣任务. 每次实验进行之前, 每个被试都需要进行 练习实验，并填写自编的实验任务理解问卷，以测量 被试对实验任务的理解情况. 确保被试理解实验内容 无误后，再进行正式实验. 每个正式实验任务完成后， 被试完成相应自编任务评估问卷，及两个测量相应个 体差异的问卷(详见5.2 (3)).

正式实验中, 每个任务共包含 130 个试次, 分为 3 个 block进行, 每个block之间有约 $1 \mathrm{~min}$ 的休息时间. 两个 任务中的每一个试次都是以时长为 $1 \mathrm{~s}$ 的注视点开始. 呈现两个选项后，被试需要在 $8 \mathrm{~s}$ 的时间内作出选择. 在 $1 \mathrm{~s}$ 的反馈之后，呈现2 6 $\mathrm{s}$ 的黑屏. 为排除任务间的 相互影响，任务之间的施测时间相隔约1 2周，任务先 后顺序随机. 两个任务的唯一差异在于, 使用不同的指 导语和反馈的内容. 实验流程参见图 1 .
除指导语和反馈外，自主跨期任务和基线折扣任 务所呈现的视觉材料完全相同, 均要求被试在两个选 项之间进行选择. 其中，基线折扣任务的指导语为: “在每一个方案中, 您都可以在一定时间后, 获得一定 数额的金钱投资. 假设用单利计算, 所有投资方案的月 利率均为 $10 \%$. 请您在看到两个选项后, 通过按键选择 在较远的时间点，本息和(本金+利息)更多的那个投资 方案”. 如果被试选择了右边的选项, 反馈为“您投资在 2 个月后获得本息和 $¥ 736$ ”(图1).

自主跨期任务的指导语为: “每一个游戏中，屏幕 的左右两边会呈现两个不同的选项，分别代表两种获 得金钱方案. 在每一个方案中，您都可以在一定时间 后, 获得一定数额的金钱. 请您在看到两个选项后, 通 过按键选择您个人更加偏好的方案”. 如果被试选择了 右边的选项，反馈为 “您选择在 2 个月后获得金额 ¥736”(图1).

为保证被试能够认真完成实验, 实验前告知被试, 每个实验结束后，从被试所做的选择中随机抽取一个, 以被试的实际选择(按照一定的比例)作为实验报酬的 一部分, 与参与实验费用合计后支付. 实验结束后, 主 试向被试解释了实验目的, 并统一支付每个被试100元

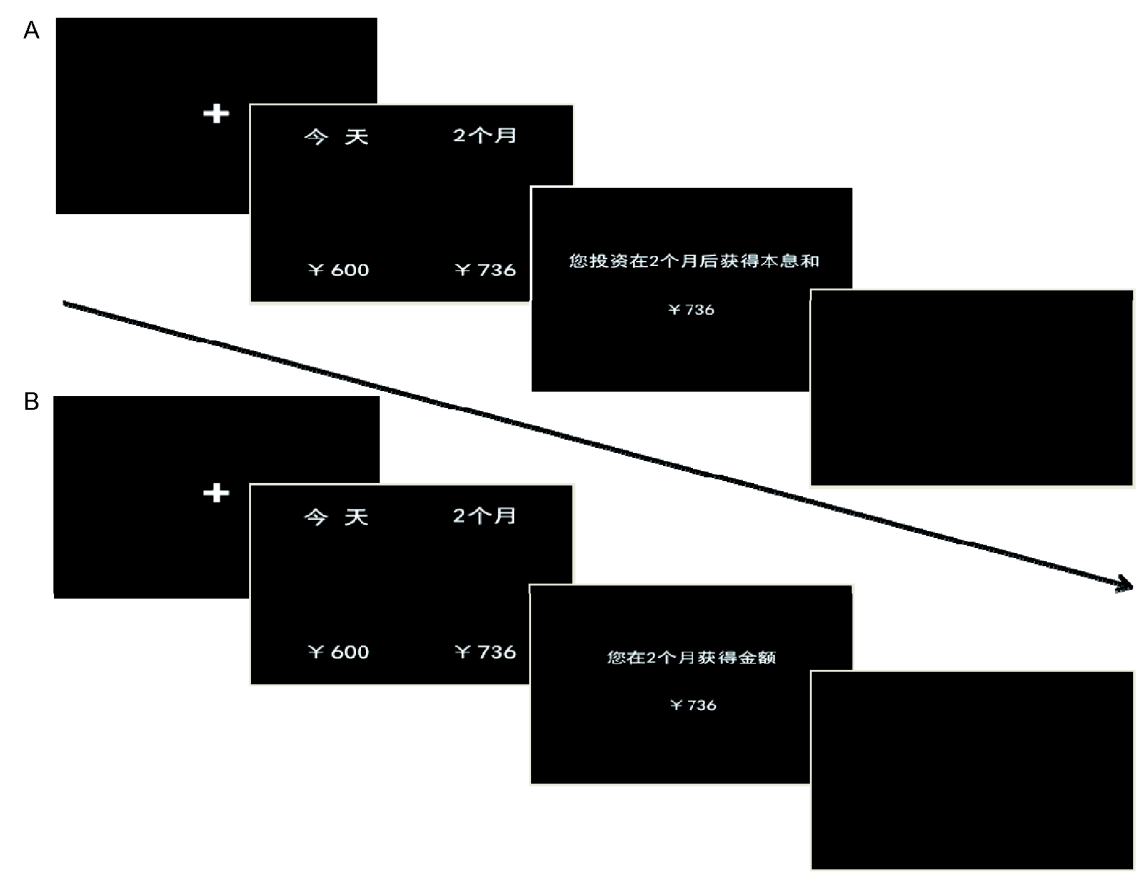

图 1 任务流程图

$\mathrm{A}$ : 基线折扣任务; $\mathrm{B}$ : 自主跨期任务 


\begin{tabular}{|c|c|c|c|}
\hline & & \multicolumn{2}{|c|}{ 结果大小 } \\
\hline & & 小 & 大 \\
\hline \multirow{2}{*}{ 计算难度 } & 高 & $\begin{array}{l}\text { SS选项: 今天, } ¥ 610 \\
\text { LL选项: } 2 \text { 个月, } ¥ 733\end{array}$ & $\begin{array}{l}\text { SS选项: 今天, } ¥ 6100 \\
\text { LL选项: } 2 \text { 个月, } ¥ 7330\end{array}$ \\
\hline & 低 & $\begin{array}{l}\text { SS选项: 今天, } ¥ 600 \\
\text { LL选项: } 2 \text { 个月, } ¥ 723\end{array}$ & $\begin{array}{l}\text { SS选项: 今天, } ¥ 6000 \\
\text { LL选项: } 2 \text { 个月, } ¥ 7230\end{array}$ \\
\hline
\end{tabular}

报酬.

（2）实验材料. 自主跨期任务和基线折扣任务所 呈现的视觉材料完全相同, 均要求被试在两个选项之 间进行选择. 在实验材料内操纵了选项的计算难度和 结果大小. 计算难度通过操纵选项金额的非零数字的 个数(如 $¥ 50$ 和 $¥ 51$ ) 来实现: 按照 $\mathrm{SS}$ 选项结果中包含 非零数字的个数, 将选项的计算难度分为计算难度低 和计算难度高两个条件. 对结果大小的操纵通过让选 项结果按照 10 倍比例增大来实现: 按照选项的结果为 百位数或千位数, 将选项的结果分为大结果和小结果 两个条件(示例见表2).

正式实验材料中，结果的延迟时间为 1 个月或 2 个 月, 小/大结果条件下, 选项结果的金额范围为 100 886 元或1000 8850元. 为了促进被试进行精确计算, 匹配 了SS和LL选项结果的折扣效用, 将二者控制在相差 1 3元(小结果条件)或者10 70元(大结果条件). 为便于 被试进行口算, 将时间折扣率设置为 0.1 , 并按照公式 1 匹配了LL选项结果的折扣效用. 此外, 控制刺激材料 约为正式刺激材料的 $10 \%$ ，但控制刺激的延迟时间范 围在1 9个月. 实验材料中SS选项和LL选项随机呈现 在屏幕中的左右位置，选项的时间在上方，结果在下 方. 所有刺激材料出现的顺序随机.

(3) 问卷. 问卷部分在实验任务完成后进行. 基线 折扣任务后被试完成基线折扣任务评估问卷和韦氏成 人智力量表算术分测验 ${ }^{[6,37]}$. 自主跨期任务后被试完 成自主跨期任务评估问卷和认知反思风格测验 ${ }^{[34]}$.

实验任务评估问卷: 问卷为自编, 主要测试被试对 实验任务的完成情况. 自主跨期任务包含了 5 个条目. 其中，3个条目测量被试基于直觉作出选择的程度、 基于计算后的结果作出选择的程度和相信按照实验说 明获得相应报酬的程度, 每个条目采用 7 级评分, 1 为 “非常不符合”, 7 为“非常符合”; 另外 2 个条目分别测量 被试报告根据计算和直觉作出选择的比例, 范围为
$0 \sim 100 \%$. 基线折扣任务比自主跨期任务多一个条目, 测量被试报告根据实验指导语中要求进行的计算方式 作出选择的比例, 范围为 $0 \sim 100 \%$.

韦氏成人智力量表算术分测验：测量被试的计算 能力. 版本为中国修订版 ${ }^{[36]}$. 该量表共14题, 由一名主 试单独施测, 最高分为 18 分. 得分越高, 表示计算能力 越高.

认知反思风格测验：测量被试认知反思风格的类 型. 该量表包含 3 个题目 ${ }^{[34]}$. 每一个题目有两个答案: 基于反思的、较缓慢的类型 $\mathrm{I}$, 和迅速得出结论而非 谨慎思考的类型 II. 例如, “若一个球拍和一个球总计 为 1.1 元, 并且球拍比球贵 1 元. 那么球值多少钱?”. 类 型 II 的答案是 10 分钱, 但这是错误答案; 基于类型 $I$ 的 答案是 50 分, 这是正确答案. 因此每个题目都需要被试 进行分析以克服最初的直觉, 经过深思熟虑后计算出 类型 I 的答案. 每个题目中, 根据被试的回答符合哪 个类型的答案, 则相应的认知反思风格的类型为 1 , 另 一个认知反思风格类型为 0 , 如果都不符合, 则该题目 的认知反思风格的两个类型得分均计为 0 . 类型 I 和类 型 II 的总分在 0 和 3 之间.

\section{6 结果}

\section{1 操作检验}

为检验实验任务范式的操作是否成功, 分析了实 验任务评估问卷的结果. 被试完成自主跨期任务和基 线折扣任务后，报告自己相信会根据指导语获得相应 报酬的程度分别为 $5.40 \pm 1.35,5.73 \pm 0.98$ ，二者显著大 于4分 $(P s<0.001)$, 说明被试相信任务的真实性. 更重 要的是, 在基线折扣任务中, 被试报告依据计算及指 导语计算方式的比例 $(79.55 \% \pm 18.96 \%)$ 显著大于其报 告根据直觉作出选择的比例 $(16.70 \% \pm 14.66 \%), t(29)=$ $11.10, P<0.001$; 但在自主跨期任务中, 被试报告自己 
根据直觉作出选择的比例 $(61.60 \% \pm 31.77 \%)$ 与其根据 计算作出选择的比例 $(40.40 \% \pm 32.45 \%)$ 无显著差异, $t$ $(29)=1.84, P=0.08$. 且被试报告, 与在自主跨期任务中 $(40.40 \% \pm 32.45 \%)$ 相比，自己在基线折扣任务中 $(79.55 \% \pm 18.96 \%)$ 更多地根据计算做出选择， $t(29)=$ $5.47, P<0.001$. 这些结果说明, 研究对两个实验任务的 操作有效，在基线折扣任务中被试更多地根据计算进 行选择，但在自主跨期任务中，被试可能并非根据计 算作出选择.

\section{2 反应时}

首先对数据进行了预处理. 自主跨期任务和基线 折扣任务共包含 6720 个试次, 删除了被试未作出选择 的试次 35 个(平均每个被试1.9个试次), 删除反应时大 于该任务平均反应时 3 个标准差或者小于 $200 \mathrm{~ms}$ 的试 次64个, 最后有效试次数为6621个. Kolmogorov-Smirnov检验表明, 自主跨期任务的反应时 $(D(30)=0.13$, $P=0.20)$ 和基线折扣任务的反应时 $(D(30)=0.11$, $P=0.20$ )均符合正态分布.

为检验假设 $\mathrm{H} 1 \mathrm{a}$ 和 $\mathrm{H} 1 \mathrm{~b}$, 将被试的反应时作为因变 量, 进行了任务类型 (自主跨期任务、基线折扣任 务 $) \times$ 计算难度 $($ 高、低 $) \times$ 结果(大、小)的三因素重复测 量方差分析(ANOVA).

结果发现，任务类型、计算难度和结果因素的主 效应显著, $F_{\text {任务 }}(1,29)=102.44, P_{\text {任务 }}<0.001, \eta^{2}$ 任务 $=0.779$, $F_{\text {难度 }}(1,29)=191.11, P_{\text {难度 }}<0.001, \eta_{\text {难度 }}^{2}=0.868, F_{\text {结果 }}(1,29)$ $=7.70, P_{\text {结果 }}=0.01, \eta_{\text {结果 }}^{2}=0.779$. 且任务类型与计算难度 的交互效应显著, $F_{\text {任务傩度 }}(1,29)=149.72, P<0.001$, $\eta^{2}=0.838$. Bonferroni事后比较发现，基线折扣任务的 反应时 $(\bar{x}=3.74 \mathrm{~s}, \mathrm{SE}=0.13)$ 要显著大于自主跨期任务 $(\bar{x}=2.26 \mathrm{~s}, \mathrm{SE}=0.11), P<0.001$ (图2). 这支持假设H1a, 说明基线折扣任务的认知加工过程比自主跨期任务更 复杂, 需要更多的反应时.

为了考察计算难度对两个任务反应时的影响, Bonferroni事后比较发现，在基线折扣任务中，高计算 难度条件下的反应时 $(\bar{x}=4.36 \mathrm{~s}, \mathrm{SE}=0.16)$ 显著大于低 计算难度条件 $(\bar{x}=3.12 \mathrm{~s}, \mathrm{SE}=0.11), P<0.001$; 在自主跨 期任务中，高计算难度条件下的反应时 $(\bar{x}=2.31 \mathrm{~s}$, $\mathrm{SE}=0.12)$ 显著大于低计算难度条件 $(\bar{x}=2.21 \mathrm{~s}, \mathrm{SE}=0.11)$, $P<0.001$ (图2). 这说明计算难度影响两个任务的反应 时, 计算难度越高, 反应时越长.
为进一步比较计算难度对两任务中反应时的影响 差异, 以高低计算难度条件下反应时之差为自变量进 行分析，发现计算难度对基线折扣任务反应时的影响 $(\bar{x}=1.23 \mathrm{~s}, \mathrm{SE}=0.09)$ 显著高于其对自主跨期任务反应 时的影响 $(\bar{x}=0.11 \mathrm{~s}, \mathrm{SE}=0.03), F(1,29)=149.72$, $P<0.001, \eta^{2}=0.838$. 这支持了假设 $\mathrm{H} 1 \mathrm{~b}$, 即自主跨期任 务的反应时与基线折扣任务有差异，计算难度对基线 折扣任务中反应时的影响大于其对自主跨期任务中反 应时的影响.

此外, 发现了另外一个并未做出预期假设的结果, 任务类型与结果的交互效应显著, $F_{\text {任务姞果 }}(1,29)=5.00$, $P=0.033, \eta^{2}=0.147$. Bonferroni事后比较发现, 在基线折 扣任务中, 大结果条件下的反应时 $(\bar{x}=3.83 \mathrm{~s}, \mathrm{SE}=0.14)$ 显著大于小结果条件 $(\bar{x}=3.65 \mathrm{~s}, \mathrm{SE}=0.12)$; 但在自主跨 期任务中二者没有显著差异, $\bar{x}_{\text {大结果 }}=2.27 \mathrm{~s}, \mathrm{SE}_{\text {大结果 }}$ $=0.12, \bar{x}_{\text {小结果 }}=2.25 \mathrm{~s}, \mathrm{SE}_{\text {小结果 }}=0.11, P=0.76$. 这说明, 结 果大小仅影响基线折扣任务的反应时: 结果越大, 反应 时越长, 但不影响自主跨期任务的反应时.

总之, 反应时分析的结果说明假设 $\mathrm{H} 1 \mathrm{a}$ 和假设 $\mathrm{H} 1 \mathrm{~b}$ 均成立. 基线折扣任务反应时的分析结果和计算难度 对其反应时的影响共同表明，基线折扣任务符合折扣 计算假设; 自主跨期任务反应时的分析结果和计算难 度对两任务的影响共同表明，自主跨期任务不同于基 线折扣任务, 其认知加工过程在复杂程度上弱于基线 折扣任务.

\section{3 选择结果}

（1）基线折扣任务的正确率. 计算了基线折扣任务 的正确率，即经过准确计算后选择出获利更多选项的 比例: 每个试次中如果被试择了获利较多的选项, 计 为 1 , 否则为 0 .

将每个被试的正确率作为因变量, 进行计算难度 (高、低) $\times$ 结果 (大、小)的二因素重复测量方差分析 (ANOVA). 结果发现计算难度的主效应显著(图3). 低 计算难度条件下的正确率 $(\bar{x}=0.92, \mathrm{SE}=0.02)$ 显著高于 高计算难度条件 $(\bar{x}=0.75, \mathrm{SE}=0.02), \quad F(1,29)=71.84$, $P<0.001, \eta^{2}=0.712$. 结果支持实验假设 $\mathrm{H} 2 \mathrm{a}$, 计算难度 越高, 基线折扣任务中的选择正确率就越低, 表明基线 折扣任务符合折扣计算假设的预期.

(2) 自主跨期任务的选择偏好. 在自主跨期任务 中, 以选择 $\mathrm{SS}$ 选项的比例作为因变量, 进行计算难度 

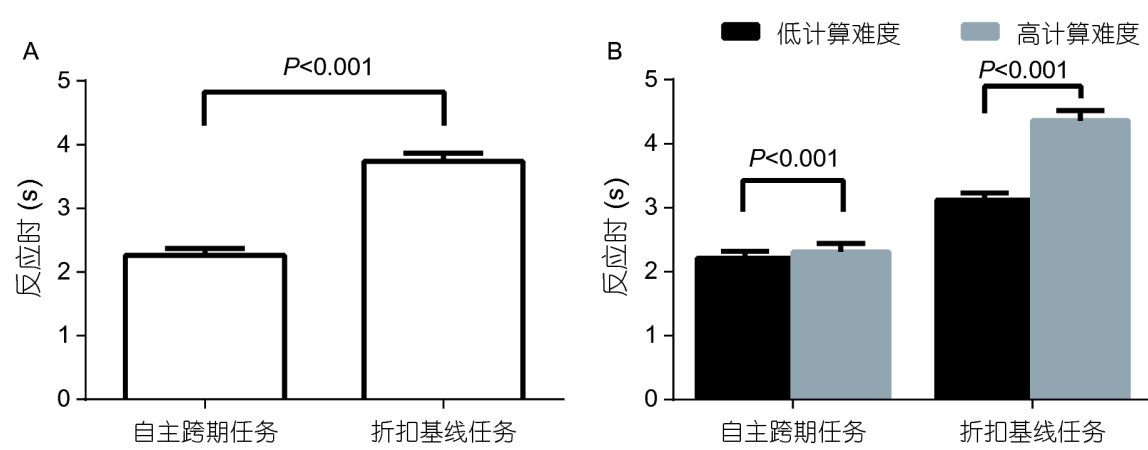

图 2 自主跨期任务和基线折扣任务的反应时 $(\bar{x} \pm \mathrm{SE})$

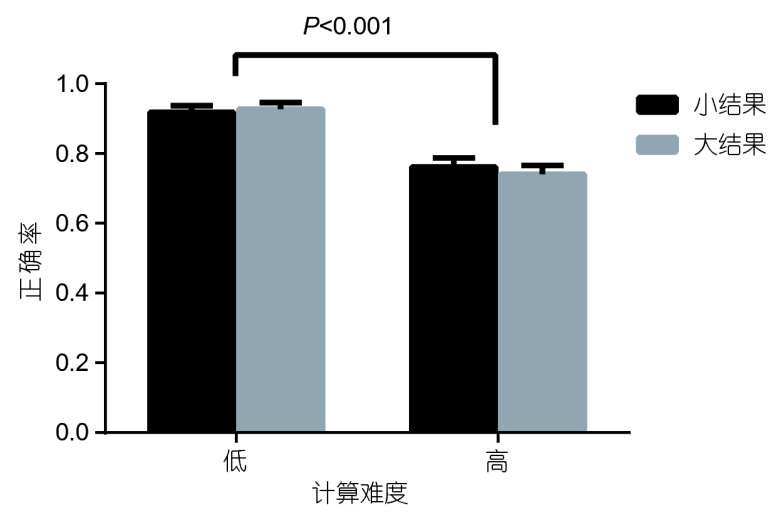

图 3 基线折扣任务的正确率 $(\bar{x} \pm \mathrm{SE})$

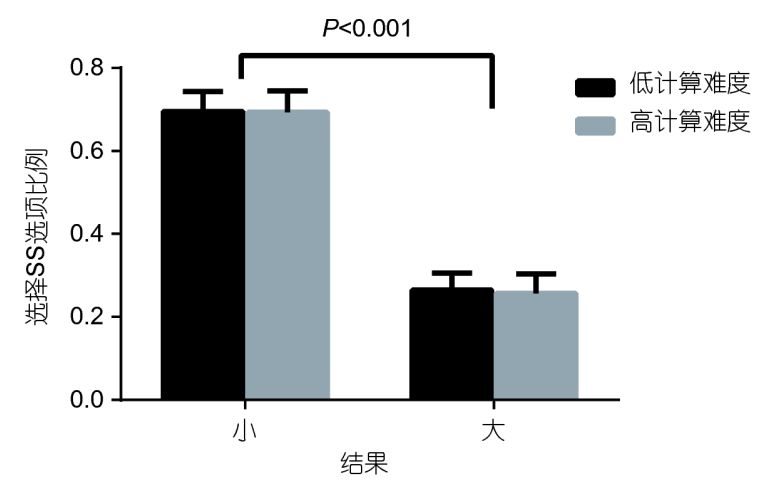

图 4 自主跨期任务中选择 $\mathrm{SS}$ 选项的比例 $(\bar{x} \pm \mathrm{SE})$

(高、低) $\times$ 结果 $(大 、 小)$ 的二因素重复测量方差分析 (ANOVA). 发现结果的主效应显著(图4). 小结果条件 下选择 $\mathrm{SS}$ 选项的比例 $(\bar{x}=0.69, \mathrm{SE}=0.05)$ 显著高于大结 果条件 $(\bar{x}=0.26, \mathrm{SE}=0.04), F(1,29)=93.57, P<0.001$, $\eta^{2}=0.763$. 这支持了假设 $\mathrm{H} 2 \mathrm{~b}$, 自主跨期任务中的选择 SS选项的比例受到选项结果大小的影响: 结果越大, 被试选择SS选项的比例越小, 更有耐心.
（3）调节变量. 为了进一步检验自主跨期任务与 基线折扣任务之间的差异，分析了计算能力和认知反 思风格个体差异变量对两个任务的作用. 调节变量和 分组后描述性统计见表 3 . 其中, 认知反思风格与计算 能力之间无显著相关, $r=-0.234, P=0.21$. 调节变量分 组后, 高低计算能力组间得分差异显著, $F(1,28)=$ 41.01, $P<0.001, \eta^{2}=0.59$; 高低认知反思风格组间得分 差异显著, $F(1,28)=86.40, P<0.001, \eta^{2}=0.75$. 说明两个 调节变量无关, 且分组有效.

计算能力. 为了检验计算能力的调节作用, 分别在 基线折扣任务和自主跨期任务中，以计算能力为协变 量, 以选择反应时或正确率/选择SS选项比例为因变量 进行了计算难度(高、低) $\times$ 结果(大、小)的协方差分析 (ANCOVA). 对反应时的分析未发现计算能力的显著 效应. 但对选择结果的分析发现，在基线折扣任务中 计算能力的主效应显著, $F(1,28)=4.18, P=0.05$, $\eta^{2}=0.130$. 将被试按照计算能力的平均数分为两组后 (表3)进行事后比较发现, 高计算能力组的被试选择正 确率 $(\bar{x}=0.88, \mathrm{SE}=0.02)$ 显著高于低计算能力组 $(\bar{x}=0.79$, $\mathrm{SE}=0.02), P=0.009$. 但在自主跨期任务中没有发现计 算能力的主效应或者交互效应. 这支持了假设H3a, 个 体的计算能力影响其基线折扣任务的绩效：个体的计 算能力越高，在基线折扣任务中的正确率越高，且计 算能力不影响其自主跨期任务的绩效.

认知反思风格. 为了检验认知反思风格的调节作 用, 分别在基线折扣任务和自主跨期任务中, 以基于 直觉的认知反思风格为协变量, 进行了类似的计算难 度 (高、低) $\times$ 结果(大、小)的协方差分析(ANCOVA). 对反应时的分析未发现认知反思风格的显著效应. 但 对选择结果的分析发现, 在自主跨期任务中认知反思 
表 3 调节变量的描述性统计

\begin{tabular}{cccc}
\hline 调节变量 & 样本 $M \pm S D$ & 高分组 $M \pm S D(n)$ & 1 低分组 $M \pm S D(n)$ \\
\hline 计算能力 & $15.27 \pm 2.18$ & $17.00 \pm 1.00(16)$ & $14.00 \pm 2.00(14)$ \\
认知反思风格(直觉) & $0.30 \pm 0.70$ & $2.00 \pm 1.00(6)$ & $0 \pm 0(24)$ \\
\hline
\end{tabular}

风格与结果大小的交互作用达到边缘显著水平, $F$ $(1,28)=3.91, P=0.06, \eta^{2}=0.123$. 将被试按照认知反思风 格的平均数分为两组后(表3)进行事后比较, 在小结果 条件下, 高得分组的选择 S S 选项比例 $(\bar{x}=0.88$, $\mathrm{SE}=0.10)$ 显著高于低分组 $(\bar{x}=0.65, \mathrm{SE}=0.05), P=0.05$; 但在大结果条件下, 高得分组的选择 $\mathrm{SS}$ 选项比例 $(\bar{x}=0.25, \mathrm{SE}=0.10)$ 与低分组 $(\bar{x}=0.26, \mathrm{SE}=0.05)$ 没有显 著差异, $P=0.10$. 在基线折扣任务中没有发现认知反 思风格的显著主效应或者交互效应。这支持了假设 $\mathrm{H} 3 \mathrm{~b}$ ，个体的认知反思风格影响其自主跨期任务的绩 效：在小金额的跨期决策中，个体的认知反思风格越 偏向直觉, 被试选择SS选项的比例就越高. 且认知反 思风格不影响其基线折扣任务的绩效.

\section{4 分层贝叶斯模型比较}

此外, 使用分层贝叶斯模型从模型拟合层面比较 了自主跨期任务和基线折扣任务的异同. 具体而言, 采用了两个最常用的跨期决策折扣模型——折扣效用 模型和双曲线折扣模型——对两个任务进行模型拟合 和参数估计, 并通过比较拟合模型的LOOIC (leaveone-out information criterion)指数确定哪个模型的拟 合度更优：LOOIC指数越低，表明模型的拟合度越 好 ${ }^{[38]}$. 随后, 通过比较所估计出的模型参数(时间折扣 率)的差异，确定两个任务是否存在显著差异. 对于不 同条件下时间折扣率，如果其后验概率分布的 $95 \%$ 最 高密度区间(highest density interval, HDI)不包含 0 , 说 明两个条件下时间折扣率存在显著差异. 使用了 $\mathrm{R}$ 软 件包hBayesDM (hierarchical Bayesian modeling of decision-making tasks) ${ }^{[22]}$ 完成了此部分的分析.

为了检验假设 $\mathrm{H} 4 \mathrm{a}$ ，使用两个折扣模型分别对基 线折扣任务和自主跨期任务进行了模型拟合，结果发 现双曲线模型对两个任务的拟合均优于折扣效用模 型：对于基线折扣任务，双曲线模型拟合的LOOIC指 数(3554)低于折扣效用模型拟合的LOOIC指数(3826), 考虑到二者LOOIC指数差异的 $95 \%$ 置信区间为 $[176$, 368 ], 该区间不包含 0 , 说明双曲线模型更符合基线折
扣任务，这也支持了基线折扣任务符合折扣计算过程 的假设; 对于自主跨期任务, 双曲线模型拟合的 LOOIC指数(3594) 明显低于折扣效用模型拟合的 LOOIC指数(2010570), 说明双曲线模型更符合基线折 扣任务. 尽管难以直接定量比较两个不同任务的模型 拟合的LOOIC指数 ${ }^{[22,39,40]}$, 但双曲线模型对基线折扣 任务的拟合稍优于自主跨期任务 (差异为 40). 随后, 进 一步比较了两个任务中拟合后的时间折扣率, 发现对 于两任务中时间折扣率之差，其后验概率分布的 $95 \%$ HDI为 $[0.035,0.096]$ (图5). 该区间没有包含 0 , 说明两 个任务的时间折扣率有显著差异. 这部分支持了假设 $\mathrm{H} 4 \mathrm{a}$ ，说明使用双曲线折扣模型可以拟合基线折扣任 务，且对基线折扣任务的拟合度优于对自主跨期任务 的拟合度, 但两个任务拟合后得到的时间折扣率存在 差异.

为了检验假设 $\mathrm{H} 4 \mathrm{~b}$, 以双曲线模型为目标模型, 依 据双分离逻辑对基线折扣和自主跨期两任务的数据按 照计算难度和结果大小两个条件进行分组, 分别进行 了双曲线模型拟合. 结果发现, 计算难度影响基线折 扣任务的拟合, 且低难度条件下拟合的LOOIC指数明 显最低, 但结果大小对该任务影响不明显; 但在自主 跨期任务中, 结果大小影响该任务的模型拟合, 且大 结果条件下拟合的LOOIC指数明显最低, 但计算能力 对该任务影响不明显(表4). 该结果支持了假设 $\mathrm{H} 4 \mathrm{~b}$, 说明计算难度和结果大小对两个任务模型拟合的影响 具有双分离效应.

\section{7 讨论}

本研究设计了新的实验范式: 符合折扣家族模型 的折扣计算过程假设的基线折扣任务, 和符合个人偏 好的自主跨期任务. 通过比较决策者在两个任务中的 任务绩效, 检验了跨期决策是否基于折扣计算过程. 研究结果表明, 被试在基线折扣任务中的绩效符合折 扣计算过程的预测, 且与自主决策任务中的绩效相比, 被试在基线折扣任务中的反应时更长. 更重要的是, 两 


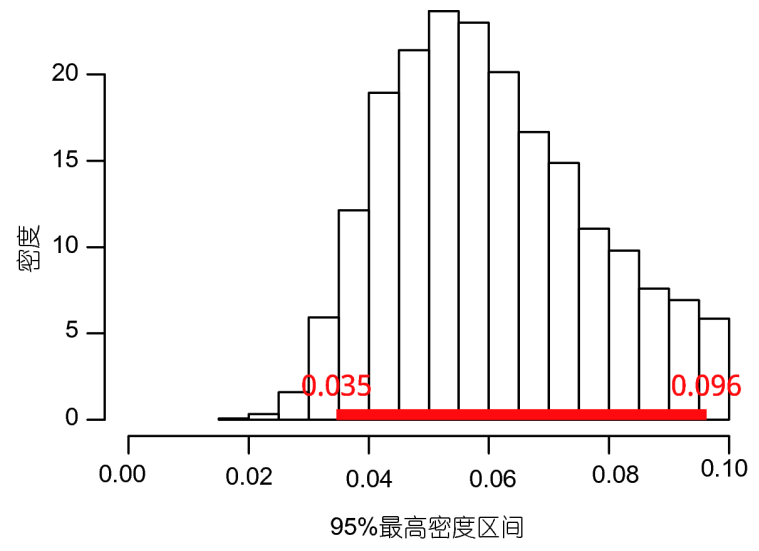

图 5 各实验任务时间折扣率之差的后验概率分布 $(95 \%$ 最 高密度区间)

个任务的绩效表现出现了双分离效应: 影响折扣计算 过程的因素，如计算难度和计算能力影响基线折扣任 务的绩效，但不影响自主跨期任务; 而与折扣计算过 程无关但与跨期决策有关的因素，如结果大小和基于 直觉的认知反思风格影响自主跨期任务的绩效, 但不 影响基线折扣任务. 此外, 模型拟合的结果表明, 双曲 线折扣模型对基线折扣任务的拟合优于自主决策任 务，且任务情境因素对模型拟合的影响出现了相似的 双分离效应：在低难度条件下，双曲线折扣模型对基 线折扣任务的拟合度更好; 而在大结果条件下, 双曲 线折扣模型对自主跨期任务的拟合度更好. 这些结果 表明，本研究设计的基线折扣任务是可靠有效的，且 符合折扣计算的假设，但被试在进行自主跨期任务时， 由于认知能力、任务特征和情境等因素的限制, 并未 遵循折扣家族模型所假设的折扣计算过程. 研究结果 也表明，基线折扣任务和自主跨期任务匹配的双分离 实验范式，可以有效地应用于跨期决策的理论模型 检验.

\section{1 基线折扣任务符合折扣计算假设}

作为基线，本研究所设计的基线折扣任务符合折
扣计算的假设，且有多个层面的证据证明其可靠有效 性. 从任务设计上看，基线折扣任务以银行投资情景 为背景, 让被试按照与双曲线折扣模型一致的银行单 利公式，通过自动加工的计算过程选择获利更高的选 项，因此其隐含的认知加工过程符合折扣计算假设. 这一假设得到了几个方面结果的支持. 从被试在任务 后的自我报告看，被试在基线折扣任务中根据计算做 出选择的比例高达 $80 \%$, 而通过直觉做出选择的比例 仅为 $16.70 \%$. 从选择结果看, 在低计算难度条件下, 被 试根据指导语进行计算然后得出正确选择的比例高达 $90 \%$, 并且在高计算难度条件下, 正确选择的比例依然 高达 $75 \%$. 从反应时看, 计算难度越高, 基线折扣任务 的反应时越高, 这符合折扣计算过程的预测, 因为计算 难度变高时, 被试为了完成计算, 需要更多的认知资 源, 因而选择时间变得更长. 从调节变量看, 个体的计 算能力越高, 基线折扣任务中经过计算选择获利较多 选项的正确率越高. 结合这些证据, 说明在基线折扣 任务中, 被试对于决策方式的自我评价、决策结果和 绩效等方面均符合折扣家族模型所假设的折扣计算 过程.

相较于以往的跨期决策模型检验范式，本研究所 设计的实验范式有更多优势. 首先，基线折扣任务克 服了以往研究中基线跨期决策任务不能自动进行折扣 计算过程的不足. 以往研究中使用的任务, 例如, Su等 人 $^{[31]} 、 Z h o u$ 等人 ${ }^{[41]}$ 和苏寅 ${ }^{[42]}$ 所设计的贴现判断任务 要求被试估算每个延迟选项等价于立即获得报酬的数 额(贴现估值), 然后选择贴现估值较大的选项. 这与折 扣模型家族所预期的折扣计算过程是一致的，通过比 较采用相同实验刺激材料的跨期决策任务与此任务比 较, 检验跨期决策的折扣计算假设. 但是贴现判断任务 并没有明确指明被试依据何种策略进行估算. 因此没 有排除掉被试依据其他非折扣计算的方式进行估算的 可能. 或者要求被试按照某一公式进行计算, 被试需要 进行充分的学习和控制加工, 才能按照任务要求做出 决策. 这样的任务设计忽略了自动加工和控制加工决

表 4 各实验条件下的双曲线模型拟合结果

\begin{tabular}{|c|c|c|c|c|}
\hline \multirow[b]{2}{*}{ 任务/条件 } & \multicolumn{2}{|c|}{ 基线折扣任务 } & \multicolumn{2}{|c|}{ 自主跨期任务 } \\
\hline & $\begin{array}{c}\text { 计算难度 } \\
\text { (低/高) }\end{array}$ & $\begin{array}{c}\text { 结果大小 } \\
\text { (小/大) }\end{array}$ & $\begin{array}{c}\text { 计算难度 } \\
\text { (低/高) }\end{array}$ & $\begin{array}{c}\text { 结果大小 } \\
\text { (小/大) }\end{array}$ \\
\hline LOOIC & $1333 / 1794$ & $1434 / 1468$ & $1828 / 1797$ & $1719 / 1313$ \\
\hline
\end{tabular}


策的差异. 但本研究中的基线折扣任务操纵被试按照 银行单利的公式进行自动加工的计算过程, 其本质与 跨期决策双曲线折扣模型的 “折扣计算” 过程一致, 操 纵检验及来自各方面的证据均证明了这一点. 其次, 本研究中的基线折扣任务可以有效地避免研究中同样 的选择结果仍可以被其他模型所解释的弊端 ${ }^{[16,43]}$. 以 往的跨期决策范式通过计算模型预测被试选择的准确 率, 或者比较不同模型的拟合度优劣, 来检验跨期决策 的认知加工过程 ${ }^{[14,21]}$. 然而, 即使某个模型具有较高的 拟合度或者预测被试选择的准确率，但是在基线折扣 任务符合折扣计算过程的基础上，通过比较自主跨期 任务与该基线任务的不同，就可以有效避免因无法明 确跨期决策过程而导致的模型检验的困难. 最后, 基 线折扣任务具有较高的生态效度. 在符合折扣计算假 设的基础上，基线折扣任务选择了基于日常生活的情 境, 即银行投资方案选择问题. 该情境贴近个体日常 生活经验, 不必强制要求被试学习, 因而也具有较高 的外部效度和生态效度.

但是，基线折扣任务并非在所有任务情境中都完 全符合折扣计算过程假设. 决策者受计算能力等因素 的限制, 不是无限理性的 ${ }^{[6]}$. 由于受到认知能力及与认 知能力相关的任务特征、参与程度等因素的影响, 个 体仍然无法完全按照折扣模型所假设的折扣计算过程 完成选择. 当计算难度增高, 基线折扣任务的反应时随 之增加; 个体计算能力越高, 被试选择的正确率也越 高. 这说明计算能力越高的被试在基线折扣任务中认 知加工能力更强, 从而有更高的比例选择出正确的选 项, 这与前人研究较为一致 ${ }^{[30,33]}$.

\section{2 检验跨期决策中的折扣计算假设}

以基线折扣任务为符合折扣计算过程的基线，检 验了自主跨期任务是否符合折扣计算假设的预测. 研 究结果发现, 自主跨期任务与基线折扣任务的绩效有 显著差异, 鉴于基线折扣任务符合折扣计算过程, 说 明被试在自主跨期任务中并没有按照折扣计算的过程 作出决策. 从反应时上看, 无论在哪种计算难度条件 下, 自主跨期任务的反应时都显著小于基线折扣任务. 从任务后自我报告看，被试报告基于计算所作选择的 比例仅为 $40.40 \%$, 远低于基线折扣任务, 并且被试在 自主跨期任务中报告基于直觉选择的比例为 $61.60 \%$, 高于基于计算所作选择的比例. 这些结果说明, 被试在
自主跨期任务中，无论是反应时还是对决策方式的自 我评价上, 均不符合折扣计算的假设. 另外, 从选择结 果来看, 当选项的时间不变而选项的结果同时变大时, 被试的选择偏好也会发生反转, 会更加倾向选择LL选 项. 这些结果意味着决策者采用的或许是一个非折扣 计算的加工过程.

研究还发现，个体的自主跨期决策过程受结果大 小的影响: 选项的结果越大, 被试越倾向于选择LL选 项, 这与前人的研究结果相一致 ${ }^{[44,45]}$. 这一结果有两种 可能的解释. 按照双曲线折扣模型的价值函数, 两个选 项的结果大小按照相同倍数增长时, 经过价值函数计 算后的两个选项的价值比例会下降, 因而被试会更偏 好 LL 选项 ${ }^{[4,44]}$; 按照非折扣模型(如权衡模型)的解释, 当时间不变而结果按照相同倍数增长时, $\mathrm{SS}$ 选项的时 间优势没有发生变化, 而LL选项的结果优势变大, 因 而被试更偏好LL选项 ${ }^{[8,45]}$. 虽然难以单独通过结果大 小效应判断确定哪种模型更优，但是结合基线折扣任 务与自主跨期任务在各个层面的不同, 本课题组认为, 个体的自主跨期决策过程可能不基于折扣计算过程, 但是可能涉及结果比较的过程.

另外, 研究发现了自主跨期任务中结果大小和认 知反思风格对绩效的交互效应. 在小结果条件下, 个 体的认知反思风格越基于直觉, 越发迅速得出结论而 不是谨慎思考, 也就更加倾向尽快获得奖赏. 但是在 大结果条件下高低认知反思风格得分组的被试对于 $\mathrm{SS}$ 选项的偏好没有显著不同. 推测这可能是结果大小 的效应较强 $\left(F(1,28)=95.53, P<0.001, \eta^{2}=0.77\right)$, 覆盖了 偏小的认知反思风格效应 $\left(\eta^{2}=0.04\right)$ 所致. 由于小金额 条件下被试对金钱奖赏的敏感性偏低, 个体更多地依 赖直觉进行跨期选择; 但是在大金额条件下, 个体对 于金钱的敏感性提高, 会更多地依赖理性的计算, 因 此没有出现认知反思风格的效应. 另一方面可能是由 于本样本中认知反思风格测验分数分布不够正态. 本 研究样本为中国大学生被试, 在基础教育阶段频繁地 进行数学测验, 导致认知反思风格得分的分布非常极 端(得分0 3的比例依次为 $80 \%, 13 \%, 3 \%, 3 \%$ ), 这也可 能影响了认知反思风格的效应.

上述结果表明, 两个任务的绩效表现, 在操作变量 和个体差异变量层面均出现了双分离效应. 这表明基 线折扣任务和自主跨期任务匹配的这一实验范式, 可 以有效地应用于跨期决策的理论模型检验中. 被试在 
进行自主跨期任务时，由于认知能力、任务特征和情 境等因素的限制，可能并未遵循折扣家族模型所假设 的折扣计算过程.

\section{3 折扣计算假设的模型拟合}

相比于传统的最大似然比估计法，分层贝叶斯模 型可以提供更为精确的假设推断. Vincent ${ }^{[23]}$ 比较了最 大似然比估计法和分层贝叶斯模型在常见的延迟折扣 任务(delay discounting task)中的优劣. 最大似然比估 计法是获得延迟折扣任务的数据后，先进行参数估计 (parameter estimation), 随后结合其他的被试信息(如 性别、实验条件等)进行假设检验. 但是被试的时间折 扣率本身会受到其他因素的影响, 如数据样本大小、 被试个体差异等. 分层贝叶斯模型可以很好地解决上 述难题, 而且可以将参数估计和假设检验结合起来, 同时被试层面的信息仍能得以保留 ${ }^{[23]}$.

通过分层贝叶斯模型拟合，结果进一步印证基线 折扣任务符合折扣计算假设. 相对于折扣效用模型, 双曲线折扣模型更符合基线折扣任务，这也与基线折 扣任务的任务本质相一致. 从基线折扣任务的模型拟 合结果看，在低计算难度条件下，双曲线折扣模型的 拟合度最优，而在高计算难度条件下，双曲线折扣模 型的拟合度最低. 考虑到基线折扣任务明确要求被试 按照银行单利公式进行相应的计算, 因此最为合理的 解释就是在低计算难度条件下，依据公式进行计算所 需要的认知加工资源较少, 因而进行折扣计算更容易, 从而使得模型拟合度在低计算难度条件下更好. 结合 任务层面、调节变量的结果，以上结果进一步证明基 线折扣任务范式的有效性.

需要注意的是，虽然本研究发现自主跨期任务的 模型拟合在大结果条件下最优，但并不能说明自主跨 期任务此时更加符合折扣计算假设. 由于目前使用的 $\mathrm{R}$ 软件包hBayesDM ${ }^{[22]}$ 只提供了跨期决策中的折扣效 用模型和双曲线折扣模型，因此无法检验并比较折扣 模型和其他非折扣模型对于自主跨期任务的拟合度. 因此，自主跨期任务更加符合何种跨期决策模型的预 测, 仍然需要后续研究进一步探索.

\section{4 研究不足与展望}

本研究存在以下 4 点不足. ( i ) 没有基于决策过程
的角度对跨期决策的折扣计算假设进行充分探讨. 常 用的决策过程追踪技术如眼动追踪技术(eye tracking) 和磁共振成像(functional magnetic resonance imaging, fMRI) 早已被大量地运用于决策过程以及认知功能的 研究 $[20,31,41,46$ - 48]. 本研究范式虽然可以确定自主跨期任 务与具有明确计算过程的基线折扣任务不同，但是仍 然无法排除自主跨期任务可能是基于 “内隐”的折扣计 算过程. 这一潜在解释可以利用过程追踪技术得到检 验. (ii) 本研究选择的反映个体差异的调节变量可能 不够敏感或者不适合于中国的大学生被试群体，如没 有发现认知反思量表在较大金额情况下的效应。未来 研究可以选取其他的调节变量, 如自我控制倾向、概 率能力等, 利用双分离逻辑进一步检验跨期决策的折 扣计算假设. (iii) 没有回答自主折扣任务更符合什么 模型的问题. 虽然本研究回答了自主跨期任务并不符 合折扣计算假设，但是没有回答该任务符合哪种决策 过程或者模型（iv) 由于实验任务本身性质的限制, 对各个任务的选择结果仅做了单方面的假设. 选择SS 选项的比例对于基线折扣任务没有意义，而选择的正 确率对于自主跨期任务没有意义. 这些问题还需要在 未来研究中做进一步的改进或者探讨.

本研究设计的研究范式与前人的研究相比, 具有 一定的传承和发展. 首先, 本研究中的基线折扣任务 在前人的研究基础上有了新的突破. 本研究所设计的 基线折扣任务要求被试按照双曲线折扣公式进行计算 并选择获利最大选项, 明确了折扣计算的过程, 从而更 有利于对跨期决策的认知加工过程进行直接检验. 其 次, 本研究在此任务范式的基础上, 使用双分离逻辑 进一步检验决策过程. 本研究所选取的操作变量计算难度和结果大小, 以及反映个体差异的调节变 量一一计算能力和认知反思风格量表, 成功地检验了 自主跨期任务和基线折扣任务的异同. 最后，在模型 拟合方面，使用能够有效避免最大似然估计法缺陷的 分层贝叶斯模型, 结合此实验范式和双分离逻辑, 为 跨期决策的折扣计算假设提供了更加充分的证据.

\section{8 结论}

研究结果发现, 从认知行为指标上看, 基线折扣任 务是符合折扣计算过程的. 无论是在哪种计算难度条 件下，基于折扣计算的基线折扣任务的反应时显著长 
于自主跨期任务. 在低计算难度条件下, 基线折扣任务 中的被试经过计算选择正确率和折扣模型的拟合度显 著高于高难度计算条件. 这些结果说明, 本研究中所设 计的基线折扣任务是有效的.

结果还表明，自主跨期任务与基线折扣任务在认 知行为指标和模型拟合层面存在不同. 在自主跨期任 务中, 反映认知加工深度的反应时短于基线折扣任务; 反映个体冲动性的选择SS选项比例, 只受到结果大小 的影响，而不受到计算难度的影响. 个体差异变量对
于自主跨期任务和基线折扣任务的影响，出现了双分 离效应: 个体的计算能力只影响基线折扣任务，不影 响自主跨期任务; 基于直觉的认知反思风格只影响自 主跨期任务, 不影响基线折扣任务. 从模型拟合层面 看，也出现了双分离效应：基线折扣任务的拟合度在 低计算难度条件下最高, 而自主跨期任务的拟合度在 大结果条件下最高. 这些结果说明，与基于折扣计算 的基线折扣任务不同，自主跨期任务抑或不是基于折 扣计算的.

致谢感谢中国科学院大学心理学系吴斌和蔡晓红为本研究收集部分数据, 感谢德国汉堡大学医学院系统神经科学系 张否对本研究的数据分析提供宝贵意见.

\section{参考文献}

1 Frederick S, Loewenstein G, O'donoghue T. Time discounting and time preference: a critical review. J Econ Lit, 2002, 40: 351-401

2 Ashraf N, Camerer C F, Loewenstein G. Adam Smith, behavioral economist. J Econ Perspect, 2005, 19: 131-145

3 Samuelson P A. A note on measurement of utility. Rev Econ Stud, 1937, 4: 155-161

4 Loewenstein G, Prelec D. Anomalies in intertemporal choice: evidence and an interpretation. Q J Econ, 1992, 107: 573-597

5 Laibson D. Golden eggs and hyperbolic discounting. Q J Econ, 1997, 112: 443-478

6 Simon H A. Information-Processing Theory of Human Problem Solving. Hillsdale: Erlbaum, 1978. 271-295

7 Read D. Intertemporal Choice. Oxford: Blackwell, 2004. 424-443

8 Scholten M, Read D. The psychology of intertemporal tradeoffs. Psychol Rev, 2010, 117: 925-944

9 Li S. A behavioral choice model when computational ability matters. Appl Intell, 2004, 20: 147-163

10 Rao L L, Li S. New paradoxes in intertemporal choice. Judgm Decis Mak, 2011, 6: 122-129

11 李纾. 决策心理: 齐当别之道. 上海: 华东师范大学出版社/“十三五”国家重点出版规划, 2016

12 Trope Y, Liberman N. Temporal construal. Psychol Rev, 2003, 110: 403-421

13 孙红月, 江程铭. 跨期决策是基于选项还是基于维度? 心理科学进展, 2016, 24: 431-437

14 Ericson K M, White J M, Laibson D, et al. Money earlier or later? Simple heuristics explain intertemporal choices better than delay discounting does. Psychol Sci, 2015, 26: 826-833 Scheibehenne B, Pachur T. Using Bayesian hierarchical parameter estimation to assess the generalizability of cognitive models of choice. Psychon Bull Rev, 2015, 22: 391-407

16 Weber E U, Johnson E J. Mindful judgment and decision making. Annu Rev Psychol, 2009, 60: 53-85

17 Savage L J. The Foundations of Statistics. New York: Wiley, 1954 van der Pol M, Cairns J. A comparison of the discounted utility model and hyperbolic discounting models in the case of social and private intertemporal preferences for health. J Econ Behav Organ, 2002, 49: 79-96

19 李纾, 毕研玲, 梁竹苑, 等. 无限理性还是有限理性? ——齐当别抉择模型在经济行为中的应用. 管理评论, 2009, 21: 103-114

20 汪祚军, 李纾. 对整合模型和占优启发式模型的检验:基于信息加工过程的眼动研究证据. 心理学报, 2012, 44: 179-198

21 Stevens J R. Intertemporal similarity: discounting as a last resort. J Behav Decis Making, 2016, 29: 12-24 Ahn W-Y, Haines N, Zhang L. Revealing neuro-computational mechanisms of reinforcement learning and decision-making with the hBayesDM package. 2016, bioRxiv:064287

23 Vincent B T. Hierarchical Bayesian estimation and hypothesis testing for delay discounting tasks. Behav Res Methods, 2016, 48: 1608-1620

24 Mazur J E. Tests of an equivalence rule for fixed and variable reinforcer delays. J Exp Psychol Anim B, 1984, 10: 426-436 

$503-507$

\section{Learn, 2013, 39: 1765-1780}

\section{5, arXiv:1507.04544} hierarchical Bayesian modeling. Front Psychol, 2016, 7: 755

40 Chavez M E, Villalobos E, Baroja J L, et al. Hierarchical Bayesian modeling of intertemporal choice. Judgm Decis Mak, 2017, 12: 19-28 Zhou L, Zhang Y Y, Li S, et al. New paradigms for the old question: challenging the expectation rule held by risky decision-making theories. J Pac Rim Psychol, 2018, 12: E17

42 苏寅. 人类决策是否遵循补偿性模型所预期的加权求和计算过程?多属性决策、风险决策、跨期决策的心理机制探索. 博士学位论文. 北 京: 中国科学院大学, 2013

43 Johnson E J, Schulte-Mecklenbeck M, Willemsen M C. Process models deserve process data: comment on Brandstätter, Gigerenzer, and Hertwig (2006). Psychol Rev, 2008, 115: 263-272

44 Lempert K M, Phelps E A. The malleability of intertemporal choice. Trends Cogn Sci, 2016, 20: 64-74 438

Zhou L, Zhang Y Y, Wang Z J, et al. A scanpath analysis of the risky decision-making process. J Behav Decis Making, 2016, 29: 169-182

骆清铭. 脑空间信息学——连接脑科学与类脑人工智能的桥梁. 中国科学: 生命科学, 2017, 47: 1015-1024

薛莉, 陶玲, 孙金洋, 等. 基于脑结构子网络分析的䇯叶肿瘤患者认知功能研究. 中国科学: 生命科学, 2017, 47: 770 


\title{
Is intertemporal decision-making based on a temporal discounting process? Evidence from double-dissociation paradigm
}

\author{
ZHANG Yang-Yang ${ }^{1}$, ZHOU Lei ${ }^{2,3,4}$, YOU XuQun ${ }^{1}$, LI Shu ${ }^{2,3}$ \& LIANG Zhu-Yuan ${ }^{2,3}$ \\ 1 School of Psychology, Shaanxi Normal University, Xi'an 710062, China; \\ 2 CAS Key Laboratory of Behavioral Science, Institute of Psychology, Beijing 100101, China; \\ 3 Department of Psychology, University of Chinese Academy of Sciences, Beijing 100049, China; \\ 4 School of Management, Jinan University, Guangzhou 510632, China
}

Intertemporal decision-making (IDM) refers to tradeoffs among options occurring at different time points. The mainstream theories of IDM can be divided into two categories according to the theories' basic hypothesis on cognitive processes, namely, whether IDM is grounded on a discounting computation process or not. The two categories are discounting and non-discounting models. However, due to the lack of reliable and effective experimental paradigms, only a few studies have directly examined whether this hypothesis is true or not. To determine if IDM is based on the discounting computation process, this study designed a new double-dissociation experimental paradigm that includes a pair of tasks, namely, a baseline discounting task, in which participants make choices by following the discounting computation process, and the free-choice intertemporal task, in which participants make choices on their own. By adopting a double-dissociation logic, this study selected a pair of manipulating variables (computational difficulty and magnitude of outcomes) and moderator variables (arithmetic ability and cognitive reflection ability) that affect only the baseline discounting task or free-choice intertemporal task. Subsequently, task performances and the hierarchical Bayesian modeling results of the pair of tasks were compared at the level of main tasks and manipulating and moderator variables. Results showed that the performance of the baseline discounting task was in line with the hypothesis of discounting computation. As computational difficulty increased, participants required increased reaction time to complete the baseline discounting task, and their task accuracy was affected by computational difficulty and arithmetic ability. The performance for the free-choice intertemporal task differed from that for the baseline discounting task. Compared with the scenario for the baseline discounting task, participants required less time to complete the free-choice intertemporal task, and they selected less sooner and smaller (SS) options when the outcome magnitude was large. Moreover, the results of moderator variables and model fitting exhibited double dissociation effects. Arithmetic ability only influenced the participants' choice in the baseline discounting task, and cognitive reflection ability only influenced the participants' preference in the free-choice intertemporal task. Meanwhile, computational difficulty affected only the model fitting for the baseline discounting task, and magnitude of outcomes affected only the model fitting in the free-choice intertemporal task. The results indicated that the new experimental paradigm was effective in testing the discounting computation process hypothesis of intertemporal choice. Furthermore, the free-choice intertemporal task might be not in line with the prediction of the discounting computation hypothesis on task performances, moderator variables, and model fitting. This study highlights a new methodological exploration for research on the intertemporal choice process.

intertemporal decision-making, discounting computation, baseline discounting task, double-dissociation, hierarchical Bayesian modeling

doi: $10.1360 / \mathrm{N} 052017-00104$ 